\title{
Targeting the Human Cerebellum with Transcranial Direct Current Stimulation to Modulate Behavior: a Meta-Analysis
}

\author{
Viola Oldrati ${ }^{1}$ - Dennis J. L. G. Schutter ${ }^{2}$ \\ Published online: 7 August 2017 \\ (C) The Author(s) 2017. This article is an open access publication
}

novel opportunities in the field of non-invasive brain stimulation to study brain-function relations [1].

Nowadays, transcranial DC stimulation (tDCS) is routinely used as a means to modulate and study functions of the cerebral cortex in the healthy and pathological brain [2]. The biophysical mechanism underlying the effects of tDCS is proposed to involve polarization of superficial nerve tissue that increases spontaneous neuronal firing rates during anodal stimulation and decrease spontaneous neural firing rates during cathodal stimulation [3-5].

Neurophysiological evidence for the ability to modulate cerebellar function using transcranial electric current comes from previous work that administered single high-voltage transcranial electric stimuli across the base of the skull and modulated activity in the dentate-thalamo-cortical pathway to M1 [6, 7]. In more recent studies, administering weak electric direct currents over the posterior fossa also showed to be effective in modulating cerebellar output $[8,9]$. For example, cerebellar tDCS has shown to interfere with motor cortex synaptic plasticity during paired associative stimulation involving median nerve and motor cortex transcranial magnetic stimulation. This finding indicates that the cerebellum may be involved in the synchronization of sensory input and motor output [9]. In another study, Galea and colleagues [8] reported polarity-dependent effects of cerebellar DC stimulation (cDCS), similar to cerebral cortical DC stimulation. Pairedpulse cerebello-cortical transcranial magnetic stimulation was used to demonstrate an increase of M1 excitability following cathodal cDCS, whereas a reduction of M1 excitability was measured after anodal cDCS. The polarity-dependent effects concur with the idea of a respective decrease and increase of Purkinje cell-mediated inhibition of M1 [8]. In further support of the physiological data, computational modeling studies have confirmed that exogenous weak electric currents at an intensity of $2 \mathrm{~mA}$ can reach the outer layers of the cerebellar 
cortex [10]. The possibility to non-invasively target the human cerebellum with tDCS introduces new opportunities to study its role in motor and also non-motor functions [11].

In spite of the available evidence, several issues that include the scalp-to-cerebellum distance, limited spatial resolution of tDCS, and the unknowns associated with the effects of exogenous direct currents at the cellular level can cause considerable variance in the extent to which DC stimulation is consistent in effectively modulating the cerebellum. Furthermore, whether cDCS has similar anodal-cathodal polarity-dependent effects as observed for DC stimulation to M1 is still an open question [8]. Particularly, since the effects of tDCS likely depend on the nature of the cerebellar process contributing to the behavior under study [12], inferences about anodal cDCS causing functional enhancement and cathodal cDCS leading to functional disruptions remain speculative. Together with the existing skepticism on the reliability of tDCS effects, we performed a metaanalysis of sham-controlled studies to examine the effects of tDCS to the cerebellum on motor and nonmotor functions in healthy volunteers. The aim of the present study was twofold: (1) Assess the efficacy and reliability of cDCS to induce behavioral effects; (2) test the hypothesis that anodal cDCS improves and cathodal cDCS impairs performance.

\section{Material and Methods}

\section{Study Selection}

A literature search was conducted using the scientific online database PubMed to identify potential studies for inclusion in the meta-analysis in the period between January 2000 and March 2017. Search criteria were "transcranial direct current stimulation" $p$ "cerebellum" and "tDCS" $p$ "cerebellum." In addition, the reference lists of previous reviews $[12,13]$ were screened to minimize the risk of overlooking potentially suitable studies for inclusion. Studies that met the following criteria were included: (i) adult healthy volunteers, (ii) shamcontrolled randomized experimental design, (iii) administration of tDCS with at least one electrode placed over the cerebellum, (iv) cognitive or motor performance index (i.e. accuracy or reaction times) as primary endpoint, (v) article published in a peer-reviewed Englishlanguage journal, and (vi) study approved by a medical ethical committees or review board. In Fig. 1, the flowchart of the selection procedure is presented.

The literature search identified a total of 184 articles. After the completion of study retrieval and removal of duplicates, title and abstract of the 96 records remained

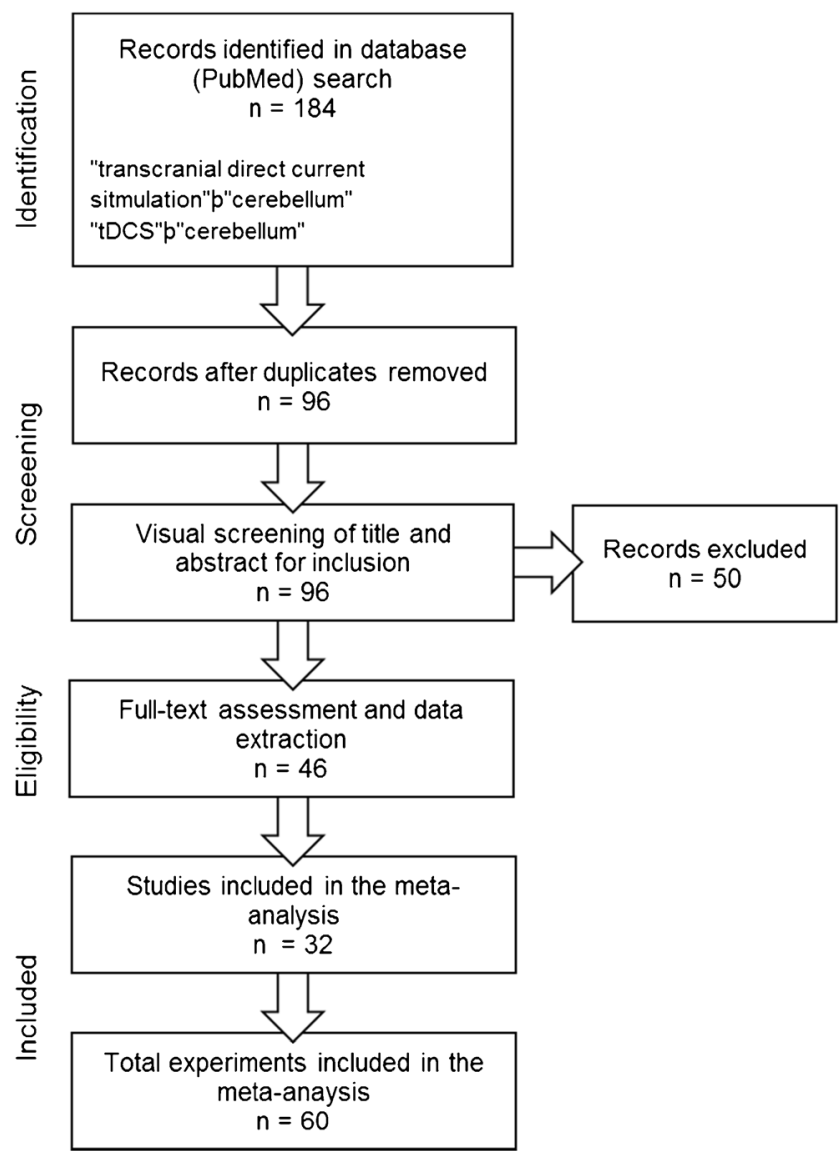

Fig. 1 Flowchart study selection procedure

were screened against inclusion/exclusion criteria. Sixty articles were selected to undergo full-text examination for eligibility, and an additional 18 studies were excluded. The remaining 32 studies were included in the present meta-analysis. Table 1 shows an overview of the main characteristics of the selected studies. Of these 32 studies, 15 studies applied both anodal and cathodal stimulation (ID: 4, 10, 14, 19, 21, 22, 24, 27, 28, 29, 31 , and 32) [17, 23, 27, 30, 32, 34-36, 39-41, 43, 44], 14 anodal (ID 1, 5, 6, 7, 8, 11, 12, 13, 15, 16, 18, 20, 23 , and 30) [14, 18-21, 24-26, 29, 31, 33, 42, 45], and three cathodal tDCS (ID: 3, 9, and 26) [16, 22, 38] (Table 1). In study 2 [15] were reported three experiments, each one comparing anodal and cathodal stimulation. Study 17 [28] includes seven experiments that applied only anodal stimulation. Study 22 [35] reports two experiments where both anodal and cathodal stimulations were used. In experiment 25 , both anodal and cathodal stimulations were applied, whereas only anodal stimulation was applied in the second experiment (ID: 22) [37]. Except for study 1 [14] that consisted of two stimulation sessions per condition, and study 4 [17] in which participants underwent three stimulation sessions 


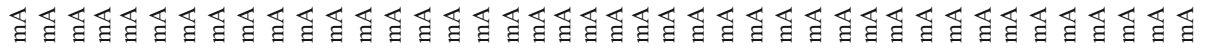

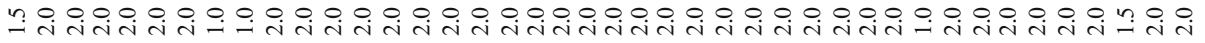

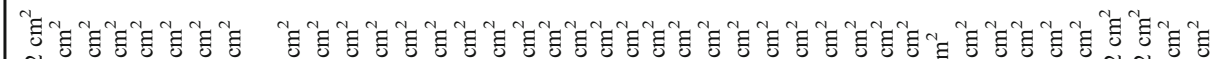

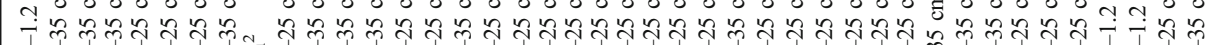

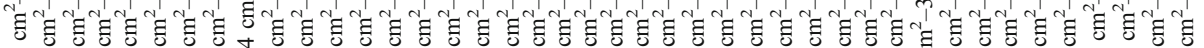

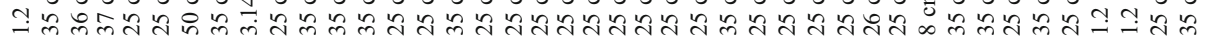

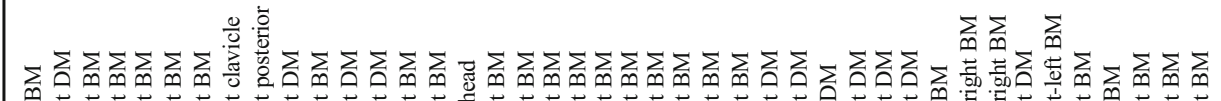
这

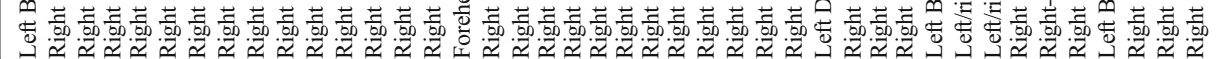

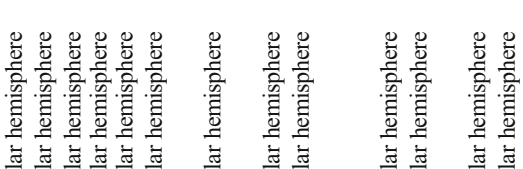

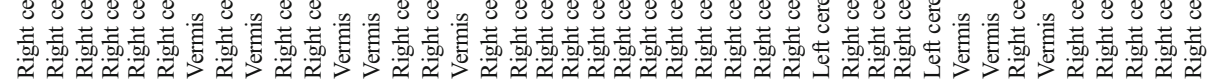

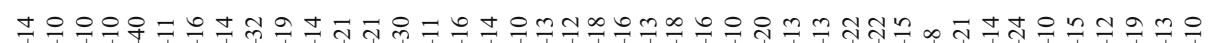

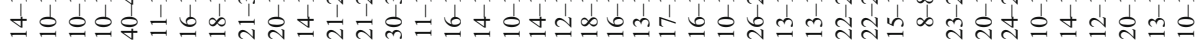




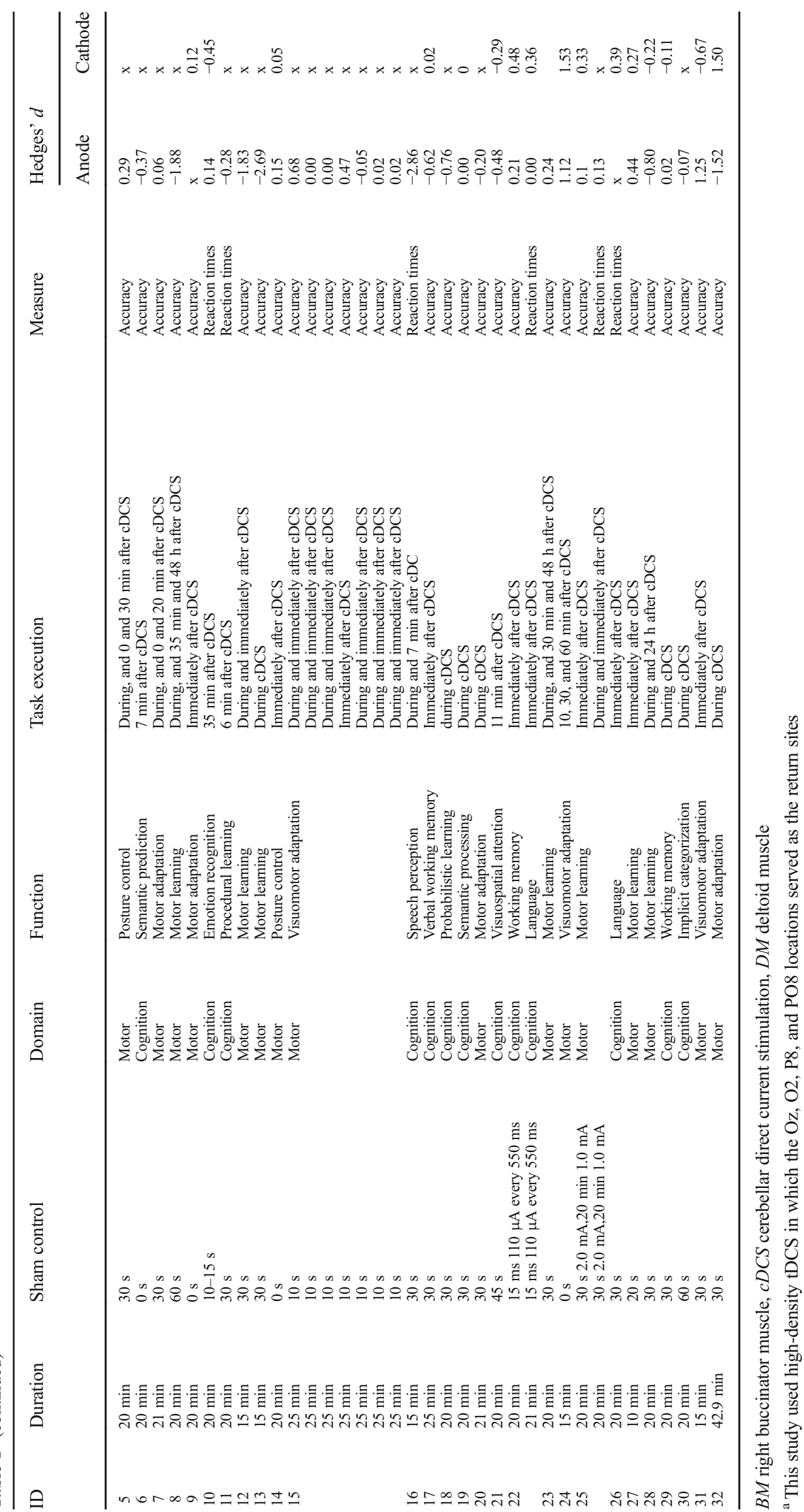


per condition, all studies administered one stimulation session per condition.

\section{Data Synthesis and Analysis}

Performance accuracy was our primary dependent variable of interest. Reaction times were used in case no data on accuracy were available. Experiments (ID: 1, 2, 4, 5, 7-12, 15-17, 1928 , and 30-32) that evaluated performance across multiple time points, the aggregate mean $\left[\left(\mu_{1}+\mu_{2}+\ldots+\mu_{n}{ }^{2}\right) / k\right]$ and pooled $\mathrm{SD}\left[\sqrt{ }\left(\left(\mathrm{SD}_{1}{ }^{2}+\mathrm{SD}_{2}{ }^{2}+\ldots+\mathrm{SD}_{\mathrm{n}}{ }^{2}\right) / k\right)\right.$, where $k=$ total number of data points, were calculated. The following descriptive data were taken from each study: sample size, mean, and standard deviation (SD) of the outcome measure for the stimulation and the sham condition. In case standard errors of the mean (SE) were provided, SD were calculated by applying the following formula $\mathrm{SD}=\mathrm{SE} \times \sqrt{ } n$. When data were presented graphically, mean and SD were estimated from the figures using free WebPlotDigitizer gms3.10 software (http://arohatgi.info/WebPlotDigitize). Corresponding authors were contacted in case the relevant numbers for the analysis could not be extracted from the paper.

The effect size metric Hedges' $d$ was used which is a standardized mean difference that accounts for the fact that the sampling variance for "active" and "sham" conditions may not always be equal [36]. From these effect sizes, the Hedges' $d$ values were calculated to correct for a bias in effect size due to small group samples [46]. For the meta-analysis, nonparametric variances were chosen to control for small sample sizes. Next, a weighted average was used to compute the cumulative effect size $(\bar{E})$ and the $95 \%$ confidence intervals $(C I)$. $\bar{E}$ represents the aggregated magnitude of the effect size of the included studies [46].

To address our first research question related to the efficacy and reliability of cDCS to induce behavioral effects, the unsigned cumulative effect sizes $(|\bar{E}|)$ were tested in a random effects model. Total heterogeneity of the effect sizes $\left(Q_{T}\right)$ was calculated and tested against the $\chi^{2}$ distribution with $(n-1)$ degrees of freedom [47]. In addition, the $I^{2}\left[\left(Q_{T}-d f /\right.\right.$ $\left.Q_{T}\right) \times 100$ ] of heterogeneity was reported which is an index for the residual proportion of the observed variance if sampling error is zero [48].

A significant $Q_{T}$ means that the variance of the effect sizes is greater than to be expected from sampling errors and suggests that the observed variance can be explained by other variables besides cDCS. To explore the robustness of the results to the possibility of publication bias, fail-safe number of studies was computed to obtain an estimate of how many nonsignificant or missing studies would render the observed metaanalytical results non-significant (Rosenthal's method: $\alpha<0.05)$. To address our second research question concerning the assumed polarity-dependent effects of cDCS, data were analyzed in a similar way as was done in the first series of analyses, except that the signed effect sizes $(\bar{E})$ were used. All analyses were performed with MetaWin version 2 [49] and Wilson's macros for meta-analyses in SPSS [50].

\section{Results}

Unsigned cumulative effect size: The random effects model ( $n=60$ ) between real and sham cDCS shows a significant $|\bar{E}|$ of $0.55,95 \% \mathrm{CI}=0.38-0.73, Z=6.27, p<0.001$. Total heterogeneity was not significant, $Q_{T}=59.74, p=0.45, I^{2}=1.23$.

Anodal cDCS showed a significant $|\bar{E}|$ of 0.59 , $95 \% \mathrm{CI}=0.34-0.83, Z=4.84, p<0.001$ (Fig. 2). Total heterogeneity was not significant, $Q_{T}=35.94, p=0.57, I^{2}=0$, and the Rosenthal's fail-safe number was 303.

Cathodal cDCS showed a significant $|\bar{E}|$ of 0.46 , $95 \% \mathrm{CI}=0.23-0.69, Z=4.23, p<0.001$ (Fig. 3 ). Total heterogeneity was not significant, $Q_{T}=23.65, p=0.26$, $I^{2}=15.43$, and the Rosenthal's fail-safe number was 131 .

Even though effect sizes of anodal and cathodal cDCS were similar, $Q=0.31, p=0.58$, cDCS was more efficacious in modulating motor, $|\bar{E}|=0.71,95 \% \mathrm{CI}=0.49-0.92$, than cognitive-related tasks, $|\bar{E}|=0.32,95 \% \mathrm{CI}=0.06-0.58$, $Q=5.24, p=0.02$. Whether performances were measured during cDCS (online), $|\bar{E}|=0.70,95 \% \mathrm{CI}=0.32-1.07$, after cDCS (offline), $|\bar{E}|=0.61,95 \% \mathrm{CI}=0.33-0.88$, or both during and after cDCS (on-offline), $|\bar{E}|=0.42,95 \% \mathrm{CI}=0.14-0.69$, did not influence the magnitude of the effect size, $Q=1.67$, $p=0.43$.

Signed cumulative effect size: The random effects model $(n=60)$ between real and sham cDCS showed a nonsignificant $|\bar{E}|$ of $0.55,95 \% \mathrm{CI}=0.38-0.73, Z=6.27$, $p<0.001$. Total heterogeneity was not significant, $Q_{T}=59.74, p=0.45, I^{2}=1.23$.

Anodal cDCS showed a non-significant $\bar{E}$ of 0.05 , $95 \% \mathrm{CI}=-0.22-0.31, Z=0.30, p=0.77$. Total heterogeneity was not significant, $Q_{T}=47.40, p=0.14, I^{2}=19.83$.

Finally, the random effects model for cathodal versus sham cDCS was not significant, $\bar{E}=0.15,95 \% \mathrm{CI}=-0.15-0.46$, $Z=1.07, p=0.29$. Total heterogeneity was also not significant, $Q_{T}=24.86, p=0.21, I^{2}=19.87$.

In sum, $1-2 \mathrm{~mA}$ cDCS is effective in modulating motorand non-motor-related performance, but there is no evidence for polarity-dependent effects of anodal and cathodal cDCS on behavioral indices of cerebellar functioning in healthy volunteers. Table 2 shows the main statistical outcomes of the meta-analysis.

\section{Discussion}

The feasibility of tDCS to modulate cerebellar functions has provided new opportunities to in vivo study cerebellar 
Fig. 2 Forest plot showing effect size estimates (Hedges' $d$ ) and 95\% confidence interval of the experiments comparing anodal with sham cDCS in healthy volunteers

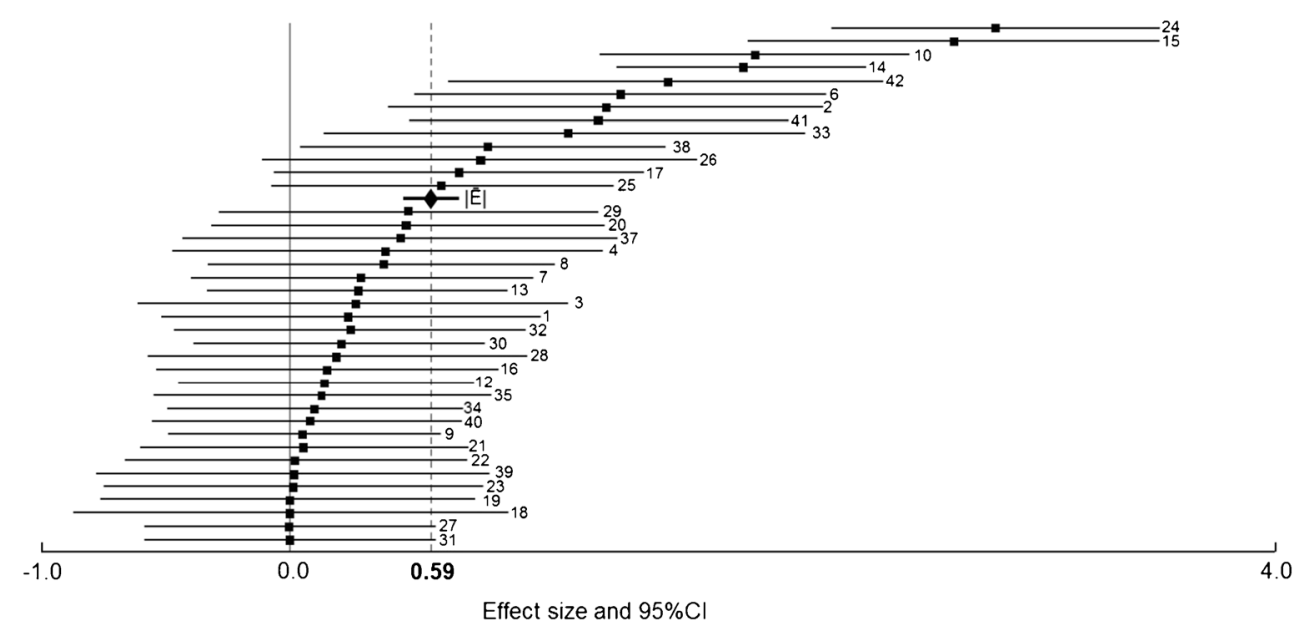

contributions in motor and non-motor-related function in healthy volunteers. In this study, we found that 1-2 mA of tDCS targeting the cerebellum is able to modulate cognitive and motor performance in healthy volunteers. Evidence for polarity-dependent effects of anodal and cathodal cDCS on improving and disrupting behavior respectively was not found.

Modeling studies suggest that the observed effects can be attributed to changes in cerebellar function. Estimations of electric field properties of bipolar DC stimulation using an inion-based cerebellum-buccinator muscle montage show that, due to the volume and homogenous structure of the cerebellum, the electric field distributions are more focused as compared to other montages targeting the cerebral cortex [43, 51]. Furthermore, lower input impedance of Purkinje cells that results in larger current flows through the cell membrane may sufficiently compensate for the low maximum electric field strength at the superficial part of the cerebellum in comparison to neurons in the cerebral cortex [10]. In line with the available neurophysiological evidence, our meta-analysis provides behavioral support for the view that weak DC can alter cerebellar functioning.
Analyses showed that motor performance was significantly more affected than cognitive performance. There may be several explanations for this finding. On one hand, this result may suggest that the cerebellum plays a more central role in motoras compared to non-motor-related functions. This is in line with the classical view of the cerebellar functions. However, our analyses do provide reliable evidence that cognitive performance is also significantly affected, indicating that the cerebellum is not exclusively related to the motor function [52-54]. A more methodologically oriented explanation for the discrepancy in effects between motor and cognitive tasks may be due to differences in the sensitivity of the task and the dependent variable of interest to detect reliable changes in performance.

Despite these converging lines of evidence, biophysical mechanisms that can account for the effects of weak static electric fields on cerebellar physiology and behavior remain elusive [5, 12]. Moreover, substantial individual variability in anatomy as well as neurophysiological constitution plays a critical role in the efficacy of cDCS. This may also in part explain why, in contrast to the popular view of polaritydependent effects of tDCS, neither anodal nor cathodal
Fig. 3 Forest plot showing absolute cumulative effect size estimates (Hedges' $d$ ) and $95 \%$ confidence interval of the experiments comparing cathodal with sham cDCS in healthy volunteers

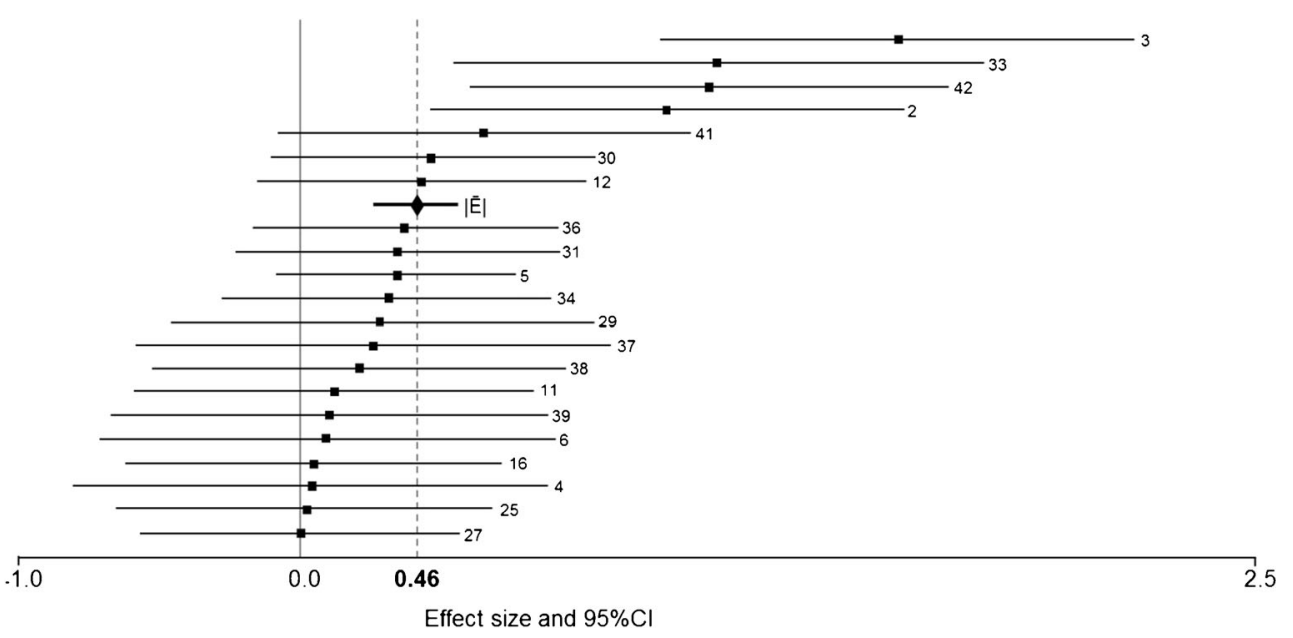


Table 2 Main results

\begin{tabular}{|c|c|c|c|c|c|c|c|c|c|c|c|c|}
\hline \multicolumn{2}{|c|}{ Random effects model } & \multirow[t]{2}{*}{ Functional domain } & \multirow[t]{2}{*}{$\mathrm{n}$} & \multirow[t]{2}{*}{ Effect size } & \multicolumn{2}{|c|}{$95 \% \mathrm{CI}$} & \multirow[t]{2}{*}{$Z$} & \multirow[t]{2}{*}{$p$ value } & \multirow[t]{2}{*}{$Q_{t}$} & \multirow[t]{2}{*}{$p$ value } & \multirow[t]{2}{*}{$I^{2}$} & \multirow[t]{2}{*}{ Fail-safe number } \\
\hline & & & & & Lower & pper & & & & & & \\
\hline \multirow[t]{5}{*}{$|\bar{E}|$} & Total & & 60 & 0.55 & 0.38 & 0.73 & 6.27 & $<0.001$ & 59.74 & 0.45 & 1.23 & 844 \\
\hline & & Motor & 37 & 0.71 & 0.49 & 0.92 & 6.49 & $<0.001$ & 33.85 & 0.57 & 0 & 330 \\
\hline & & Cognition & 23 & 0.32 & 0.06 & 0.58 & 2.39 & 0.02 & 15.13 & 0.85 & 0 & 148 \\
\hline & Anodal & & 39 & 0.59 & 0.34 & 0.83 & 4.84 & $<0.001$ & 35.94 & 0.57 & 0 & 303 \\
\hline & Cathodal & & 21 & 0.46 & 0.23 & 0.69 & 4.23 & $<0.001$ & 23.65 & 0.26 & 15.42 & 131 \\
\hline \multirow[t]{3}{*}{$\bar{E}$} & Total & & 60 & -0.09 & -0.31 & 0.13 & 0.78 & 0.44 & 63.61 & 0.32 & 7.81 & - \\
\hline & Anodal & & 39 & 0.05 & -0.22 & 0.31 & 0.30 & 0.77 & 47.40 & 0.14 & 19.83 & - \\
\hline & Cathodal & & 21 & 0.15 & -0.15 & 0.46 & 1.07 & 0.29 & 24.86 & 0.21 & 19.87 & - \\
\hline
\end{tabular}

$C I$ confidence interval, $|\bar{E}|$ unsigned cumulative effect size, $\bar{E}$ signed cumulative effect size

cDCS predicted the respective enhancement or impairment in behavior. A factor presumed to be involved may concern the unknowns regarding the degree to which the cerebellum is actively engaged in a particular motor task or cognitive function. For example, if the cerebellum does not play a role in a given neural processing stream, then anodal cDCS may increase spontaneous firing rates in the cerebellum. As a result, unrelated signals are introduced in the brain that may actually interfere with neural processing, causing functional impairments rather than improvements. In addition, the inhibitoryexcitatory nature and timing of its contributions may be important for understanding the effects of a uniform electric field on cerebellar tissue. In fact, this issue may even be more critical when cDCS is delivered simultaneously during performance. At this point, one can only speculate about the functional role of the cerebellum in non-motor-related behavior. According to the universal cerebellar transform hypothesis, the cerebellum integrates internal and external information to optimize performance according to context [55]. The integration is suggested to reflect a coordinated sequence of processes associated with thought and action that occurs in the cortico-pontine-cerebellar-thalamo-cortical circuit [55]. Electric stimulation of the cerebellum appears a feasible technique to further examine the universal cerebellar transform hypothesis. However, in order to be able to better understand and predict the behavioral effects of cDCS, unraveling the working mechanisms of the cerebellum on the molecular, structural, and system's level is required.

Even though the main outcomes of the meta-analyses support the view that DC stimulation is a viable approach to investigate cerebellar functions, the variability in outcome measures as well as the applied stimulation parameters across studies should be taken into account. In particular, our results do not unequivocally establish that cDCS is effective in all circumstances for reasons we discussed earlier. However, our data do show that low electric currents applied to the surface of the scalp can produce effects that may shed light on the contributions of the cerebellum in motor- and non-motor-related performance, but that is difficult to predict the direction of the effects relying on current polarity. Further research on how weak direct currents establish effects on cerebellar tissue and influence functional processes may help to improve cDCS designs.

Finally, several limitations of the present study should be mentioned. The limited number of studies available for our analysis prevented us from performing sub-analyses to explore questions such as under which circumstances polaritydependent effects may still be applicable to cDCS and which types of function are particularly sensitive to cDCS. Also, our findings do not provide mechanistic insights into the cerebellar workings, and adding neuroimaging techniques to future studies seems a logical next step.

In conclusion, DC stimulation is effective in modulating cerebellar function, but no support is found for polaritydependent effects of anodal and cathodal cDCS on enhancing and disrupting behavior, respectively.

\section{Compliance with Ethical Standards}

Conflict of Interest The authors declare that they have no conflict of interest.

Open Access This article is distributed under the terms of the Creative Commons Attribution 4.0 International License (http:// creativecommons.org/licenses/by/4.0/), which permits unrestricted use, distribution, and reproduction in any medium, provided you give appropriate credit to the original author(s) and the source, provide a link to the Creative Commons license, and indicate if changes were made.

\section{References}

1. Nitsche MA, Paulus W. Excitability changes induced in the human motor cortex by weak transcranial direct current stimulation. J Physiol. 2000;527:633-9. 
2. Filmer HL, Dux PE, Mattingley JB. Applications of transcranial direct current stimulation for understanding brain function. Trends Neurosci. 2014;37:742-53.

3. Nitsche MA, Cohen LG, Wassermann EM, Priori A, Lang N, Antal A, et al. Transcranial direct current stimulation: state of the art 2008 . Brain Stimul. 2008;1:206-23.

4. Shin YI, Foerster Á, Nitsche MA. Transcranial direct current stimulation (tDCS)-application in neuropsychology. Neuropsychologia. 2015;69:154-75.

5. van Dun K, Bodranghien F, Manto M, Mariën P. Targeting the cerebellum by noninvasive neurostimulation: a review. Cerebellum. 2017;16:695-741.

6. Ugawa Y, Rothwell JC, Day BL, Thompson PD, Marsden CD. Percutaneous electrical stimulation of corticospinal pathways at the level of the pyramidal decussation in humans. Ann Neurol. 1991;29:418-27.

7. Ugawa Y, Day BL, Rothwell JC, Thompson PD, Merton PA, Marsden CD. Modulation of motor cortical excitability by electrical stimulation over the cerebellum in man. J Physiol. 1991;441:57-72.

8. Galea JM, Jayaram G, Ajagbe L, Celnik P. Modulation of cerebellar excitability by polarity-specific noninvasive direct current stimulation. J Neurosci. 2009;29:9115-22.

9. Hamada M, Strigaro G, Murase N, Sadnicka A, Galea JM, Edwards MJ, et al. Cerebellar modulation of human associative plasticity. J Physiol. 2012;590:2365-74.

10. Parazzini M, Rossi E, Ferrucci R, Liorni I, Priori A, Ravazzani P. Modelling the electric field and the current density generated by cerebellar transcranial DC stimulation in humans. Clin Neurophysiol. 2014;125:577-84.

11. Tremblay S, Austin D, Hannah R, Rothwell JC. Non-invasive brain stimulation as a tool to study cerebellar-M1 interactions in humans. Cerebellum Ataxias. 2016;3:19. doi:10.1186/s40673-016-0057.

12. Van Dun K, Bodranghien F, Mariën P, Manto M. TDCS of the cerebellum: where do we stand in 2016? Technical issues and critical review of the literature Front Hum Neurosci. 2016;11:199.

13. Priori A, Ciocca M, Parazzini M, Vergari M, Ferrucci R. Transcranial cerebellar direct current stimulation and transcutaneous spinal cord direct current stimulation as innovative tools for neuroscientists. J Physiol. 2014;592:3345-69.

14. Avila E, Van Der Geest JN, Kengne Kamga S, Verhage MC, Donchin O, Frens MA. Cerebellar transcranial direct current stimulation effects on saccade adaptation. Neural Plast. 2015;2015: 968970.

15. Beyer L, Batsikadze G, Timmann D, Gerwig M. Cerebellar tDCS effects on conditioned eyeblinks using different electrode placements and stimulation protocols. Front Hum Neurosci. 2017;11:23.

16. Boehringer A, Macher K, Dukart J, Villringer A, Pleger B. Cerebellar transcranial direct current stimulation modulates verbal working memory. Brain Stimul. 2013;6:649-53.

17. Cantarero G, Spampinato D, Reis J, Ajagbe L, Thompson T, Kulkarni K, et al. Cerebellar direct current stimulation enhances on-line motor skill acquisition through an effect on accuracy. J Neurosci. 2015;35:3285-90.

18. Craig CE, Doumas M. Anodal transcranial direct current stimulation shows minimal, measure-specific effects on dynamic postural control in young and older adults: a double blind, sham-controlled study. PLoS One. 2017;12:e0170331.

19. D'Mello AM, Turkeltaub PE, Stoodley CJ. Cerebellar tDCS modulates neural circuits during semantic prediction: a combined tDCSfMRI study. J Neurosci. 2017;37:1604-13.

20. Doppelmayr M, Pixa NH, Steinberg F. Cerebellar, but not motor or parietal, high-density anodal transcranial direct current stimulation facilitates motor adaptation. J Int Neuropsychol Soc. 2016;22:1-9.

21. Ehsani F, Bakhtiary AH, Jaberzadeh S, Talimkhani A, Hajihasani A. Differential effects of primary motor cortex and cerebellar transcranial direct current stimulation on motor learning in healthy individuals: a randomized double-blind sham-controlled study. Neurosci Res. 2016;112:10-9.

22. Fernandez L, Albein-Urios N, Kirkovski M, McGinley JL, Murphy AT, Hyde C, et al. Cathodal transcranial direct current stimulation (tDCS) to the right cerebellar hemisphere affects motor adaptation during gait. Cerebellum. 2016;16:168-77.

23. Ferrucci R, Giannicola G, Rosa M, Fumagalli M, Boggio PS, Hallett $\mathrm{M}$, et al. Cerebellum and processing of negative facial emotions: cerebellar transcranial DC stimulation specifically enhances the emotional recognition of facial anger and sadness. Cogn Emot. 2012;26:786-99.

24. Ferrucci R, Brunoni AR, Parazzini M, Vergari M, Rossi E, Fumagalli M, et al. Modulating human procedural learning by cerebellar transcranial direct current stimulation. Cerebellum. 2013;12:485-92.

25. Galea JM, Vazquez A, Pasricha N, Orban De Xivry JJ, Celnik P. Dissociating the roles of the cerebellum and motor cortex during adaptive learning: the motor cortex retains what the cerebellum learns. Cereb Cortex. 2011;21:1761-70.

26. Hardwick RM, Celnik P. Cerebellar direct current stimulation enhances motor learning in older adults. Neurobiol Aging. 2014;35: 2217-21.

27. Inukai Y, Saito K, Sasaki R, Kotan S, Nakagawa M, Onishi H. Influence of transcranial direct current stimulation to the cerebellum on standing posture control. Front Hum Neurosci. 2016;10:325.

28. Jalali R, Miall RC, Galea JM. No consistent effect of cerebellar transcranial direct current stimulation (tDCS) on visuomotor adaptation. J Neurophysiol. 2017. doi:10.1152/jn.00896.2016.

29. Lametti DR, Oostwoud Wijdenes L, Bonaiuto J, Bestmann S, Rothwell JC. Cerebellar tDCS dissociates the timing of perceptual decisions from perceptual change in speech. J Neurophysiol. 2016;116:2023-32.

30. Macher K, Bohringer A, Villringer A, Pleger B. Cerebellar-parietal connections underpin phonological storage. J Neurosci. 2014;34: 5029-37.

31. Majidi N, Verhage MC, Donchin O, Holland P, Frens MA, van der Geest JN. Cerebellar tDCS does not improve performance in probabilistic classification learning. Exp Brain Res. 2016;235:421-8.

32. Miall RC, Antony J, Goldsmith-Sumner A, Harding SR, McGovern C, Winter JL. Modulation of linguistic prediction by tDCS of the right lateral cerebellum. Neuropsychologia. 2016;86:103-9.

33. Panico F, Sagliano L, Grossi D, Trojano L. Cerebellar cathodal tDCS interferes with recalibration and spatial realignment during prism adaptation procedure in healthy subjects. Brain Cogn. 2016;105:1-8.

34. Picazio S, Granata C, Caltagirone C, Petrosini L, Oliveri M. Shaping pseudoneglect with transcranial cerebellar direct current stimulation and music listening. Front Hum Neurosci. 2015;9:1-9.

35. Pope P, Miall RC. Task-specific facilitation of cognition by cathodal transcranial direct current stimulation of the cerebellum. Brain Stimul. 2012;5:84-94.

36. Shah B, Nguyen TT, Madhavan S. Polarity independent effects of cerebellar tDCS on short term ankle visuomotor learning. Brain Stimul. 2013;6:966-8.

37. Shimizu RE, Wu AD, Samra JK, Knowlton BJ. The impact of cerebellar transcranial direct current stimulations (tDCS) on learning fine-motor sequences. Philos Trans R Soc Lond Ser B Biol Sci. 2017;372:20160050.

38. Spielmann K, van der Vliet R, van de Sandt-Koenderman WM, Frens MA, Ribbers GM, Selles RW, et al. Cerebellar cathodal transcranial direct stimulation and performance on a verb generation task: a replication study. Neural Plast. 2017;2017:1254615.

39. Steiner KM, Enders A, Thier W, Batsikadze G, Ludolph N, Ilg W, et al. Cerebellar tDCS does not improve learning in a complex whole body dynamic balance task in young healthy subjects. PLoS One. 2016;11:e0163598. 
40. Taubert M, Stein T, Kreutzberg T, Stockinger C, Hecker L, Focke A, et al. Remote effects of non-invasive cerebellar stimulation on error processing in motor re-learning. Brain Stimul. 2015;9:692-9.

41. Van Wessel BW, Claire Verhage M, Holland P, Frens MA, van der Geest JN. Cerebellar tDCS does not affect performance in the Nback task. J Clin Exp Neuropsychol. 2015:38319-26.

42. Verhage MC, Avila EO, Frens MA, Donchin O, van der Geest JN. Cerebellar tDCS does not enhance performance in an implicit categorization learning task. Front Psychol. 2017;8:476.

43. Yavari F, Mahdavi S, Towhidkhah F, Ahmadi-Pajouh MA, Ekhtiari $\mathrm{H}$, Darainy M. Cerebellum as a forward but not inverse model in visuomotor adaptation task: a tDCS-based and modeling study. Exp Brain Res. 2016;234:997-1012.

44. Zuchowski ML, Timmann D, Gerwig M. Acquisition of conditioned eyeblink responses is modulated by cerebellar tDCS. Brain Stimul. 2014;7:525-31.

45. Samaei A, Ehsani F, Zoghi M, Hafez Yosephi M, Jaberzadeh S. Online and offline effects of cerebellar transcranial direct current stimulation on motor learning in healthy older adults: a randomized double-blind sham-controlled study. Eur J Neurosci. 2017;45: $1177-85$.

46. Hedges LV, Olkin I. Statistical methods for meta-analysis. 1st ed. London: Academic Press; 1985.
47. Hedges LV. Distribution theory for Glass's estimator of effect size and related estimators. J Educ Stat. 1981;6:107.

48. Borenstein M, Higgins JP, Hedges LV, Rothstein HR. Basics of meta-analysis: $\mathrm{I}^{2}$ is not an absolute measure of heterogeneity. Res Synth Methods. 2017;8:5-18.

49. Rosenberg MS, Adams DC, Gurevitch J. MetaWin: statistical software for meta-analysis version 2. Sunderland, Massachusetts: Sinauer Associates; 2000.

50. Lipsey MW, Wilson DB. Practical meta-analysis. 1ste ed. London: Sage Publications; 2001.

51. Rampersad SM, Janssen AM, Lucka F, Aydin Ü, Lanfer B, Lew S, et al. Simulating transcranial direct current stimulation with a detailed anisotropic human head model. IEEE Trans Neural Syst Rehabil Eng. 2014;22:441-52.

52. Middleton FA, Strick PL. Cerebellar projections to the prefrontal cortex of the primate. J Neurosci. 2001;21:700-12.

53. Ramnani $\mathrm{N}$. The primate cortico-cerebellar system: anatomy and function. Nat Rev Neurosci. 2006;7:511-22.

54. Stoodley CJ, Schmahmann JD. Evidence for topographic organization in the cerebellum of motor control versus cognitive and affective processing. Cortex. 2010;46:831-44.

55. Koziol LF, Budding D, Andreasen N, D'Arrigo S, Bulgheroni S, Imamizu H, et al. Consensus paper: the cerebellum's role in movement and cognition. Cerebellum. 2014;13:151-77. 